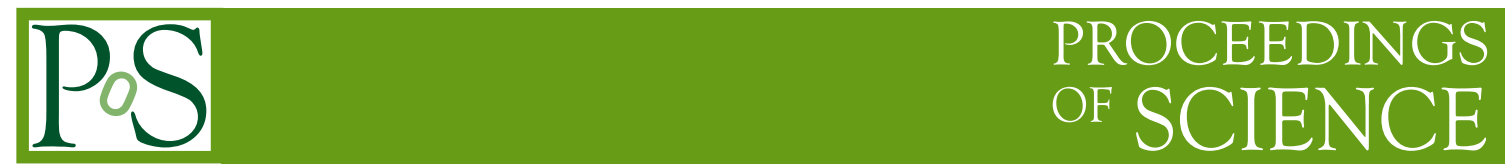

\title{
Tracking detectors for future Linear Colliders
}

\author{
Jochen Kaminski* \\ Universität Bonn \\ E-mail: kaminski@physik.uni-bonn.de
}

\begin{abstract}
The tracking detectors proposed for future linear collider experiments ILC and CLIC are designed to yield excellent momentum resolution for individual charged particles, which is necessary for the most precise reconstruction of the Higgs and other objects. Additional requirements for the tracking detectors arise from the particle flow algorithm, which promises excellent jet energy measurement. The two proposed detector concepts, $\mathrm{SiD}$ and ILD, forsee main tracking devices based on silicon layers or time-projection detector technology respectively. The requirements on these systems and the R\&D carried out to fulfill the specifications will be described.
\end{abstract}

The European Physical Society Conference on High Energy Physics

18-24 July, 2013

Stockholm, Sweden

\footnotetext{
${ }^{*}$ Speaker.
} 


\section{Two detector concepts for both linear colliders}

The International Linear Collider is a planned linear electron-positron collider complementary to the LHC. In several stages, energies between $250 \mathrm{GeV}$ and $1 \mathrm{TeV}$ are considered to cover the full physics potential of such an accelerator. The main physics target is a complete understanding of the Higgs-sector including the Higgs self-coupling and model independent measurement of the Higgs decay width. In addition, precision measurements of top-quark and electroweak gauge boson coupling constitute a major physics case. In spite of the limited center-of-mass energy, the ILC exceeds the discovery potential of the LHC for certain, mainly weakly interacting new particles. To study the particle reactions two different detectors are proposed. Both detectors [1], SiD and ILD, will be constructed to take data alternately. They will be swapped in and out of the interaction point by a push-pull principle. For the linear collider CLIC at CERN two very similar detectors are envisaged [2], but because of the higher center-of-mass energy between $500 \mathrm{GeV}$ and $3 \mathrm{TeV}$ some modifications are necessary.

The design requirements of SiD and ILD are driven by the expected physics program. In particular the Higgs recoil measurement, where two high energetic muons of a Z-decay are to be measured, gives the most stringent requirement for the tracking detectors. The transverse momentum resolution of such tracks is given by $\frac{\partial p_{t}}{p_{t}^{2}}=a \oplus \frac{b}{p_{t} \sin \theta}$, where $a$ should be as small as $a=2.0 \times 10^{-5} \frac{1}{\mathrm{GeV}}$. In Fig. 1 the influence of this parameter on the Higgs mass spectrum is shown. The overall detector design follows the requirements of the particle flow algorithm (PFA), which is proposed as the reconstruction concept for linear colliders. The PFA combines information from calorimeters and tracker to obtain the best possible jet energy reconstruction. To this end, it needs a highly efficient tracking detector, which can identify and separate charged tracks also in high track density environments. For this, the ratio $\frac{B R^{2}}{\sigma}$, where $B$ is the magnetic field, $R$ the radius of the tracker and $\sigma$ the spatial resolution, must be as high as reasonably possible.

\section{Tracking detectors at SiD}

The SiD detector concept foresees a high magnetic field of $B=5 \mathrm{~T}$ and accepts a shorter lever arm than the ILD. The main tracking device (see Fig. 2) is made of five layers of single-sided silicon micro-strip detectors. The inner layer will be at a radius $R_{i}=0.22 \mathrm{~m}$, the outermost at $R_{o}=1.22$
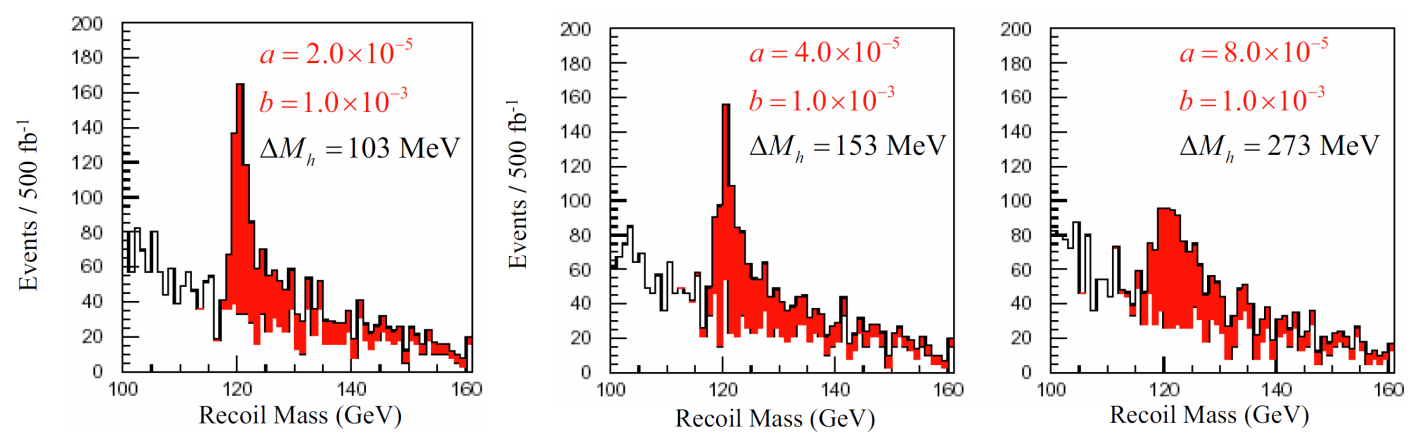

Figure 1: Higgs recoil mass spectra for several values of parameters characterizing the tracker momentum resolution [3]. 

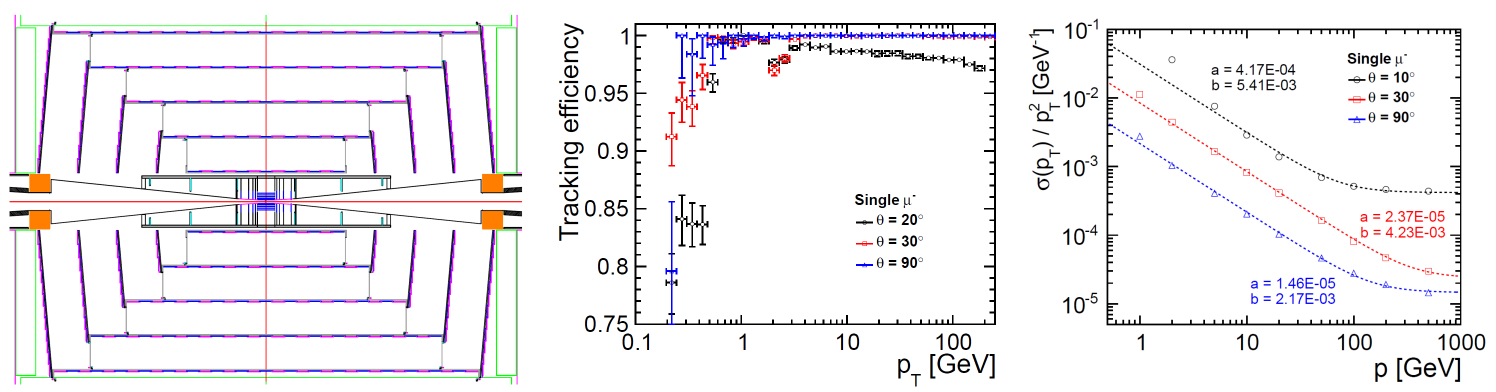

Figure 2: $\mathrm{SiD}$ tracking detector. Left side: $r-z$ view of detector layout, middle: tracking efficiency of single muons as function of the momentum, and right: transverse spatial resolution of single muons. [1]

m. To cover a large solid angle the endcaps are equipped with four conical discs with double layers of single-sided silicon micro-strip detectors. All sensors have an area of $10 \times 10 \mathrm{~cm}^{2}$, a thickness of $300 \mu \mathrm{m}$ and a strip pitch of $50 \mu \mathrm{m}$. A very challenging task is to minimize the material budget of the all-silicon tracker. To reach the goal of $0.9 \% X_{0}$ per layer, in particular the non-sensitive material is minimized as far as possible. For example, an integrated pitch adapter is used by adding on the sensor an additional metal layer, where the signals are routed from the micro-strips to the readout chip. The readout strip is bonded directly on the sensor and the service cables are made of printed circuits on kapton. The characteristic bunch structure of both linear colliders allows for reducing the material budget even further: in case of the ILC 1312 bunches with a bunch crossing time of $554 \mathrm{~ns}$ form a bunch train, which is repeated every $200 \mathrm{~ms}$, and CLIC has a similar scheme with about $20 \mathrm{~ms}$ between bunch trains. During the long intervals between bunch trains the readout electronics can be shut down reducing the energy consumption by one to two orders of magnitude down to less than $600 \mathrm{~W}$ for the complete tracker. This feature is called power pulsing and allows for cooling the detector only by air flow and omitting complex cooling systems.

The performance of the tracker was studied in simulations and based on an integrated tracking approach, where both the vertex pixel detector and the micro-strip main tracker are not used separately but are considered as an entity, an excellent track finding efficiency could be demonstrated. Also, the required momentum resolution was achieved (see Fig. 2).

\section{Tracking detectors at ILD}

The ILD concept foresees a weaker magnetic field of $B=3.5 \mathrm{~T}$, but a longer lever arm. The tracking system consists of several subdetectors of which the main tracking device is a time projection chamber (TPC) with an overall length of $4.7 \mathrm{~m}$ and a radius from $0.33 \mathrm{~m}$ to $1.8 \mathrm{~m}$. The silicon envelop covers the TPC on all sides (see Fig. 3) and is divided into three different subdetectors: the Silicon Inner Tracker (SIT), the Silicon External Tracker (SET) and the Endcap Tracker Detector (ETD). The SIT-TPC-SET combination provides redundancy in the barrel region and allows for internal calibration of the tracking. The tracking system is completed by 7 discs of the Forward Tracking Detector (FTD).

Simulation studies have shown that the tracking efficiency and the momentum resolution requirements can be reached. Fig. 3 shows for example the efficiency for $t \bar{t} \rightarrow 6$ jets and the momentum resolution for single muon events. 

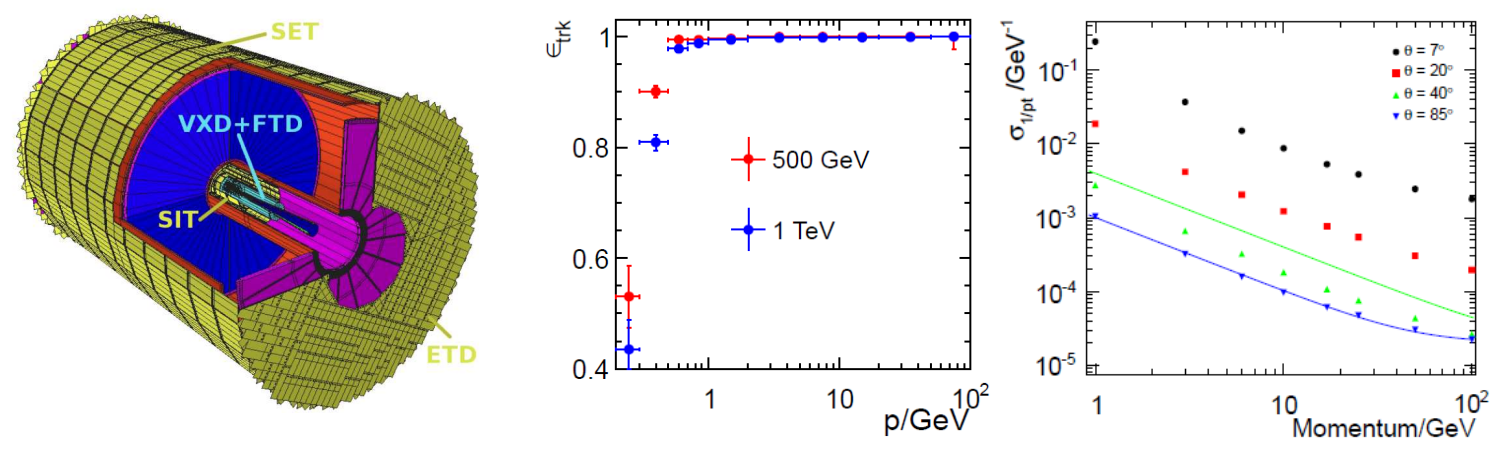

Figure 3: ILD tracking detector. Left side: 3D view of the tracking detectors, middle: tracking efficiency in $t \bar{t} \rightarrow 6$ jets events in dependence on the momentum, and right: transverse momentum resolution of single muons. The lines show $\sigma \frac{1}{p_{T}}=2 \times 10^{-5} \frac{1}{\mathrm{GeV}} \oplus \frac{1 \times 10^{-3}}{p_{T} \sin \theta}$ for $\theta=30^{\circ}$ (green) and $\theta=85^{\circ}$ (blue). [1]

\subsection{SIT, SET and ETD}

The silicon envelope is made of several layers of double sided silicon strip dectors. The SIT consists of two layers between the vertex detector and the TPC and helps to link the two subdetectors' measurements, since the time stamping of the TPC is not as well defined as the very precise vertex detector. Another tool is the 'topological time stamping' (hooking up TPC tracks to the SIT hits) which will be valuable for correct bunch-crossing association and for reducing backgrounds [1]. The SET is a single layer detector between the TPC and the electromagnetic calorimeter and gives a very precise measurement at a large lever arm. This improves the momentum resolution of the TPC by a factor of five and gives a good link between hits in the electromagnetic calorimeter and the TPC. The ETD finally is also a single layer detector covering the endcap region of the TPC and gives a precise position measurement for tracks that have few measurement points in the TPC.

The silicon envelope covers a very large area of $180 \mathrm{~m}^{2}$ with approximately $10^{7}$ channels. To facilitate the production of such a large area, the same detector layout will be used throughout the tracker but with variable strip length depending on the location. To study the design of the sensors there is a close cooperation of the LHC detector upgrade and ILD groups. For both projects flat sensors are developed, which have an integrated pitch adapter and an edgless sensor layout.

\subsection{Forward Tracking Detector}

The number of track points of TPC, SIT, SET and ETD drops to zero for tracks below $\theta \approx 15^{\circ}$ (see Fig. 4). To reach a good angular acceptance the FTD is necessary to give up to 7 additional track points down to $\theta \approx 7^{\circ}$. This gains in importance at higher center- of-mass energies, when particles are more likely to be boosted in the forward region. In particular at more than $500 \mathrm{GeV}$ the vector boson fusion dominates the Higgs production and gives highly boosted final states electrons.

\subsection{Time Projection Chamber}

The prominent feature of the TPC is the large number of track points (more than 200 at ILD), which ensures an easy background reduction and efficient track finding and enables the reconstruction of non-pointing tracks (see an example event in Fig 4). In addition the TPC has a homogeneously distributed material budget of only $0.03 X_{0}$ in the barrel region and provides a good 

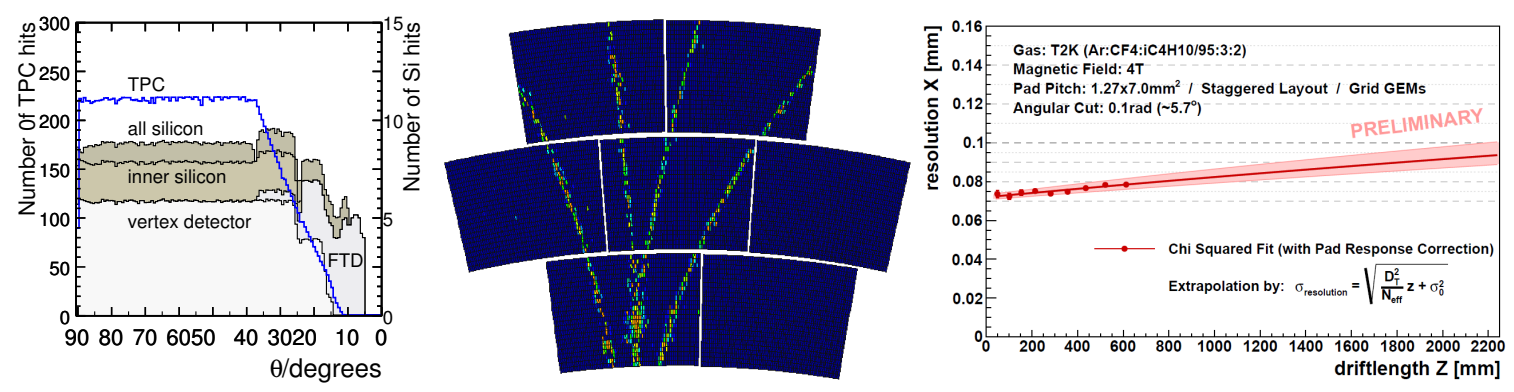

Figure 4: Left side: number of track points in dependence on the inclination $\theta$, middle: example event of a Micromegas-based TPC at the DESY test beam, and right: transverse spatial resolution of a triple GEM-based TPC in a $4 \mathrm{~T}$ magnetic field. [1]

particle identification for lower energetic tracks thanks to a $d E / d x$ measurement of $5 \%$ accurancy. In contrast to past TPCs, however, the stringent requirements on the readout can only be met by Micro-Pattern Gaseous Detectors. Currently a Micromegas- and two GEM-based designs are studied experimentally. Both gas amplification technologies have similar advantages of very small pitch between the sensitive area and thus an improved spatial resolution, fast signals and strongly reduced ion back drift. The LCTPC collaboration has studied many technical aspects of these new devices. For example first systematic studies of the ion back drift were done both for the Micromegas and the GEM and tests in high magnetic fields were performed (see spatial resolution of a triple GEM detector at $B=4 \mathrm{~T}$ in Fig. 4). Also new concepts for large area coverage were investigated. For example the use of a resistive coverage of the readout pads in combination with a Micromegas were first studied to increase the pad size and allow for a reduced number of electronic readout channels. As an interesting alternative to a pad-based readout, the use of GridPix detectors is studied, where a pixel readout chip is used as an active anode. This readout is sensitive to single primary electrons and features a very high granularity, which reduces strongly the occupancy.

\section{Summary}

Despite their different approaches on the tracking device, both the SiD and ILD experiments planned for the ILC show similar performance as far as track finding efficiency and momentum resolution is concerned. At CLIC, due to the higher background rates, the all-silicon approach performs better, see ref. [2]. Work on the technical challenges for the trackers is progressing well and first devices which can meet the physics requirements have been tested.

\section{References}

[1] Ties Behnke, et al., The International Linear Collider, Technical Design Report, Volume 4, Detectors, 2013

[2] L. Linssen, et al.,Physics and Detectors at CLIC: CLIC Conceptual Design Report. CERN, 2012.

[3] Ties Behnke, et al., International Linear Collider, Reference Design Report, Volume 4, Detectors, August 2007 